\title{
Modelos empírico-estadísticos de rendimiento de maíz en los principales estados productores de maíz de los Estados Unidos
}

\author{
Empirical-statistical models for maiz yield in the principal maiz producing states in the united \\ states
}

\author{
Julio Alfonso Arakaki Kiyan ${ }^{1}$; Juan Carlos Chang Chang Fun ${ }^{2}$
}

\begin{abstract}
Resumen
En este trabajo se buscaron modelos de regresión lineal y cuadrática múltiple para cada uno de los principales estados productores de maíz de los Estados Unidos. Se tomó como variable dependiente el rendimiento anual de maíz y como variables independientes, al año, temperaturas promedio mensuales y precipitaciones totales mensuales. Se encontró que el tamaño óptimo de la muestra de observaciones era de 30 años para todos los estados. Se consideró al mejor modelo como aquel que tuviera el coeficiente de correlación múltiple corregido más alto o la desviación estándar más baja. Los modelos, así como sus parámetros estadísticos, fueron obtenidos usando técnicas estándares de regresión múltiple. El criterio usado para validar los modelos fue el coeficiente de correlación entre los rendimientos observados y los rendimientos estimados. En base a esta prueba solamente un modelo, el del estado de Indiana, tuvo significancia estadística para estimar rendimientos de maíz a futuro.
\end{abstract}

Palabras clave: maíz; modelo agroclimático; modelo empírico-estadístico de pronóstico de cosechas; estimación maíz Estados Unidos.

\begin{abstract}
In this research, multiple lineal and quadratic regression models for leading maize producing states in the US were generated and assessed. The dependent variable was annual maize yield and the independent variables were year, average monthly temperatures and total monthly rainfall. The optimum observation sample size was 30 years for all states. We considered that the best model was the one that showed the highest adjusted multiple correlation coefficient or the lowest model standard deviation. The models, as well as their statistic parameters, were calculated using standard multiple regression techniques. The criterion used to validate the models was the correlation coefficient found between observed and estimated crop yield. Based on this test, only one model, the one from the State of Indiana, had statistical significance to estimate future maize yields.
\end{abstract}

Keywords: maize; agroclimatic model; crop prediction empiric-statistical model; maize production assessment United States.

\section{Introducción}

El maíz es uno de los tres cereales más importantes que el hombre usa para su alimentación, directa o indirectamente, como alimento procesado, o para el ganado. En el Perú, el maíz amiláceo es usado directamente para consumo humano, mientras que el maíz amarillo duro es usado, predominantemente, como fuente de alimento para aves, ganado porcino y bovino. La demanda nacional de este último no es satisfecha por lo que es necesario importar. De otro lado, Estados Unidos de Norteamérica es el principal productor de maíz y un modelo agroclimático que pronostique su producción o rendimiento sería muy útil para negociar mejores precios de importación. El presente trabajo tiene los siguientes objetivos: 1)
Obtener los mejores modelos de regresión múltiple para determinar los rendimientos de maíz en los principales estados productores de maíz de los EE.UU.; 2) Determinar el mejor tamaño de muestra para la obtención de estos modelos y 3) Determinar los modelos que puedan ser usados para pronóstico de cosecha en años posteriores a aquellos usados para generar los mismos.

\section{Materiales y métodos}

\section{Selección de los principales estados productores de} maíz

De los principales estados productores de maíz, se han descartados aquellos que tienen registros de información 
meteorológica posterior a 1987 y a aquellos cuya producción es predominantemente bajo riego, quedando seleccionados los estados de Iowa, Illinois, Minnesota, Indiana, Dakota del Sur, Ohio, Kansas, Missouri, Texas y Colorado.

\section{Información de rendimiento de maíz}

La información de rendimiento de maíz fue obtenida de la página web del Departamento de Agricultura de los EE.UU: http://www.gov/wps/portal/usdahome. En la Fig. 1 se muestra la evolución temporal del rendimiento del cultivo de maíz para el estado de Colorado.

\section{Software}

MINITAB 14 Statistical Software, para obtener los modelos de regresión y realizar el análisis estadístico.

\section{Datos climáticos}

Se usaron datos climáticos mensuales obtenidos de Monthly Climatic Data for the World, 1956-2006. Weather Bureau. S.S. Department of Commerce; ubicados en: http://www7.ncdc.noaa.gov/IPS/mcdw/mcdw.html. Para el caso específico de Indiana, los datos se obtuvieron de Climatological Data, 1940-2005, Indianápolis, Indiana (IMCPL collection), ubicados en: http://in-ulib-brayton. ads.iu.edu/climatedata/print/home.html.

\section{Obtención de los mejores modelos de regresión múltiple}

Un modelo de regresión múltiple (Molinero, 2002) es planteado de la siguiente forma:

$$
Y=a_{0}+a_{1} X_{1}+a_{2} X_{2}+\ldots a_{k} X_{k}+\varepsilon
$$

Donde $\mathrm{Y}$ es la variable dependiente, $\mathrm{X}_{1}, \mathrm{X}_{2}, \ldots$ son variables independientes o predictoras, $\mathrm{a}_{0}, \mathrm{a}_{1}, \mathrm{a}_{2} \ldots$ son coeficientes de regresión y $\varepsilon$ es el error aleatorio, $\mathrm{k}$ es el número de variables independientes.

En los modelos de regresión se consideró como variable dependiente el rendimiento anual del cultivo (en $\mathrm{kg} / \mathrm{ha}$ ) y como variables independientes al año, las temperaturas mensuales promedio del aire $\left(\mathrm{en}^{\circ} \mathrm{C}\right)$ y las precipitaciones mensuales acumuladas (en $\mathrm{mm}$ ) de los meses que dura la campaña agrícola. También se consideró a los cuadrados respectivos de estas variables climáticas.

En la mayoría de los casos se trabajó con los meses de abril a setiembre, salvo que la época de siembra en algunos de los estados empezara un mes antes o un mes después de abril.

Una vez ordenado el conjunto de datos, se procesó con el programa MiniTab 14, el cual proporcionó los modelos correspondientes. La elección del mejor modelo se hizo en base al criterio del menor valor del error residual $\mathrm{S}$, el cual es calculado de la siguiente manera (Córdova, 2008): Donde $\mathrm{n}$ es el número de observaciones, $\mathrm{k}$ es el número

$$
S=\sqrt{\frac{\sum_{i=1}^{n}\left(y_{i}-\hat{y}_{i}\right)^{2}}{n-(k+1)}}
$$

de variables regresoras (predictoras), y es el rendimiento observado e y es el rendimiento estimado con el modelo. Adicionalmente al error residual, en un buen modelo de regresión múltiple, la variancia explicada por el modelo debe ser significativamente mayor al error residual. Para determinar si la variancia explicada por el modelo es mayor al error residual se utiliza la prueba $\mathrm{F}$ (de Fisher). Este estadístico tiene una distribución de probabilidad conocida, la cual depende del valor $\mathrm{F}$ y del número de grados de libertad de las variancias que son comparadas.

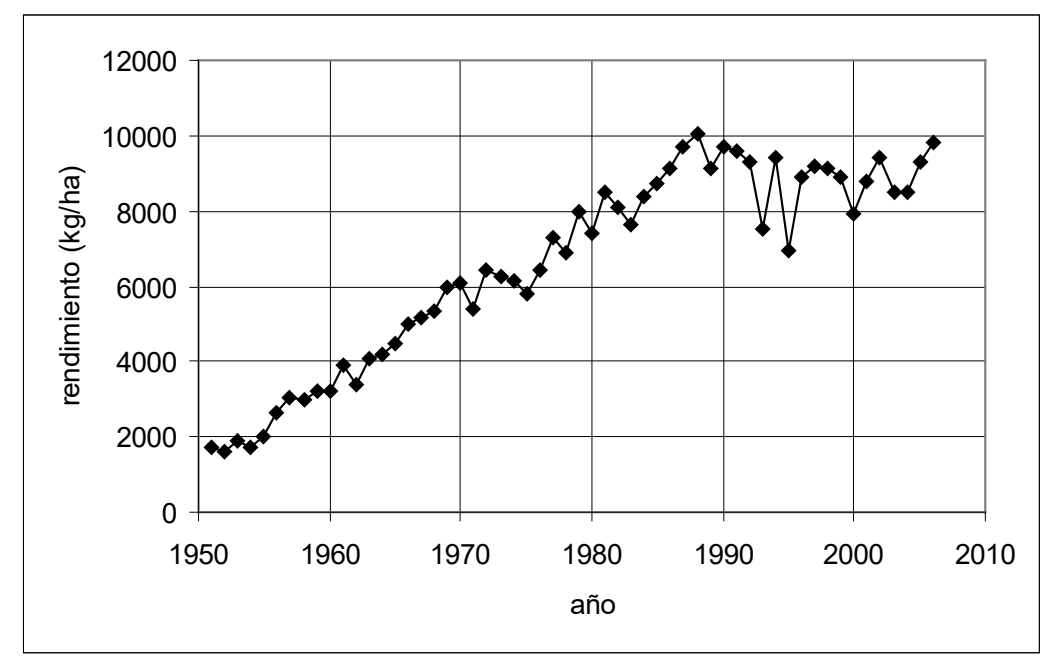

Figura 1. Evolución temporal del rendimiento de cultivo de maíz para el estado de Colorado 


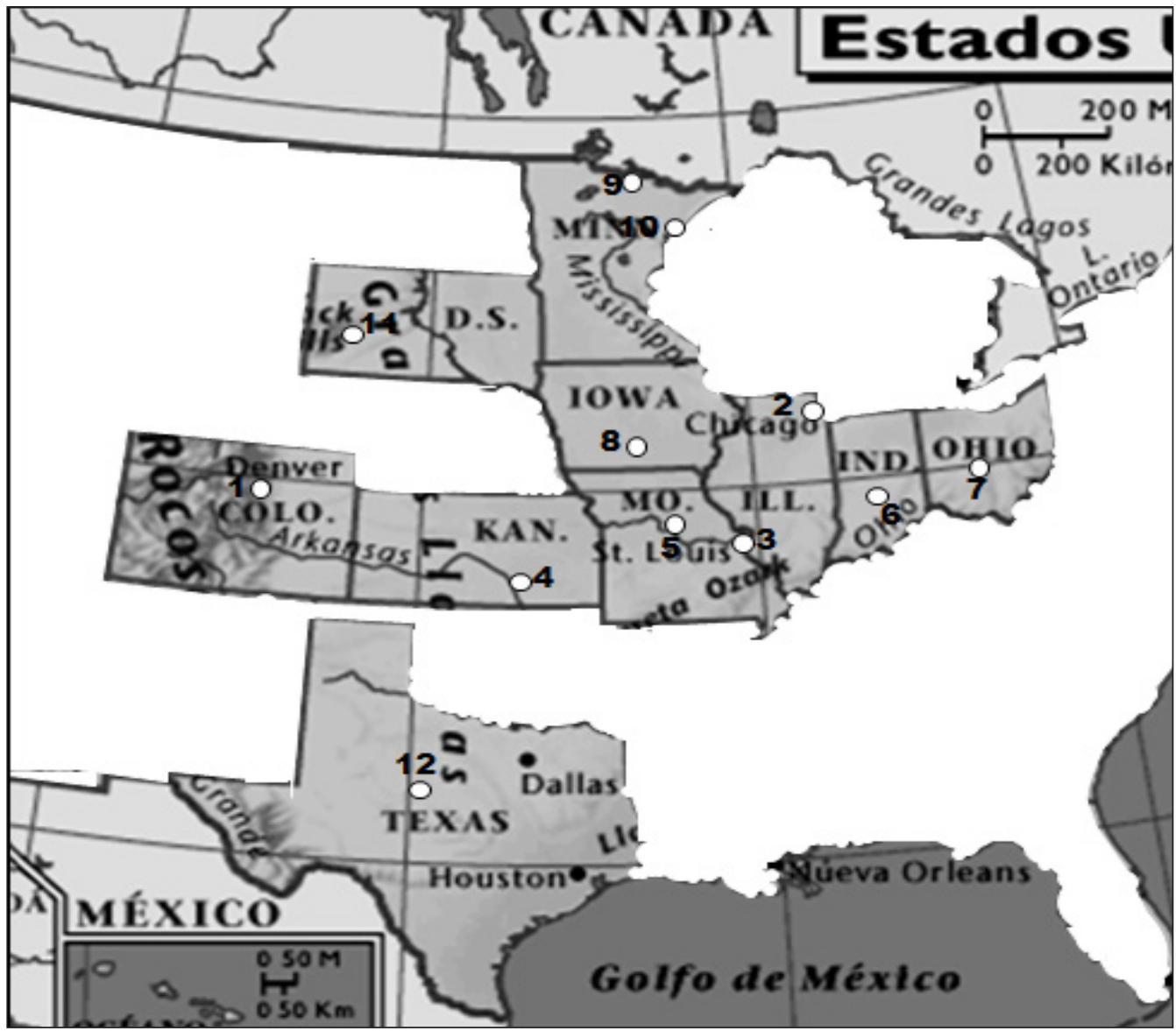

Figura 2. Mapa de los estados productores de maíz; los estados que no forman parte del estudio se han pintado de blanco. Se indica la ubicación de las estaciones meteorológicas (con un círculo blanco y numeradas) usadas para este trabajo. 1. Denver, 2. Chicago, 3. San Louis, 4. Wichita, 5. Columbia, 6. Indianapolis, 7. Columbus, 8. Des Moines, 9. International Falls, 10. Duluth, 11. Rapid City, 12. Abilene.

La variancia explicada por el modelo está dada por la siguiente ecuación:

Donde y es el promedio de los rendimientos observados. El estadístico F (calculado) es definido como:

$$
S_{\text {modelo }}^{2}=\frac{\sum_{i=1}^{n}\left(\hat{y}_{i}-\bar{y}\right)^{2}}{k}
$$

Los programas de computadora que calculan los modelos de regresión realizan un análisis de variancia a partir

$$
F=\frac{S_{\text {modelo }}^{2}}{S^{2}}
$$

del cual se puede determinar si el modelo es válido. Se considera válido un modelo en el cual la probabilidad (p) de encontrar un valor de F mayor al F calculado es menor a un valor consensuado. El valor de $\mathrm{p}$ indica la probabilidad de aceptar el modelo como válido cuando en realidad no lo es.

\section{Determinación del mejor tamaño de muestra}

Se emplearon tres tamaños de muestra: 30, 35 y 40 años, desde la década de los años 50 hasta la década de los años 90, pues en ese rango de años se tiene completa la información climática en los 10 estados de estudio. Para cada tamaño de muestra se obtuvo el mejor modelo y de los tres se seleccionó el que tenía el menor valor del error residual S. El tamaño de la muestra, cuyo modelo fue seleccionado, es el tamaño óptimo de la muestra. El proceso se repite por cada estado.

Para explicar los resultados obtenidos, se hizo la regresión lineal simple de rendimiento contra el año, en dos períodos; el primero desde 1956 a 1970 y el segundo desde 1971 a 1995, para los estados de Colorado, Texas y Kansas. Con las varianzas obtenidas del análisis de regresión (el cuadrado de la desviación $\mathrm{S}$ ), se calculó el estadístico $\mathrm{F}_{\text {año }}$ :

$$
\mathrm{F}_{\text {año }}=\frac{\operatorname{varianza}(1971-1995)}{\operatorname{varianza}(1956-1970)}
$$

Y luego, se comparó el valor calculado con el que se encuentra en Tablas. 


\section{Obtención del modelo de la muestra de tamaño óptimo}

No todas las variables independientes propuestas inicialmente se incluyeron en el modelo. Se eligió aquellas que aparecían en el mejor modelo de la muestra de tamaño óptimo. Cuando ya se determinaron las variables del modelo, el programa MiniTab les hizo un análisis de regresión para proporcionar la ecuación matemática del modelo de la muestra estudiada y los parámetros estadísticos.

\section{Selección de modelos para pronóstico de cosecha}

En base a los modelos obtenidos, se estimaron los rendimientos de maíz con datos de los cinco años posteriores a la muestra usada para construir los modelos. Posteriormente, se obtuvo el coeficiente de correlación muestral $r$ definido por Snedecor y Cochran (1980) de la regresión lineal entre el rendimiento estimado y el observado para cada estado.

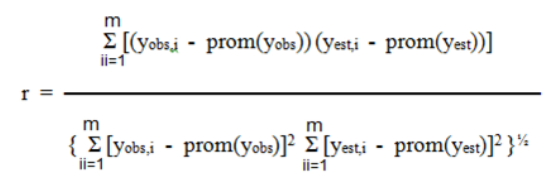

Donde $\mathrm{m}$ es el tamaño de la muestra de prueba, $\mathrm{y}_{\text {est }}$ e $\mathrm{y}_{\mathrm{obs}}$ son los rendimientos estimados y observados, respectivamente; $\operatorname{prom}\left(\mathrm{y}_{\mathrm{obs}}\right)$ y $\operatorname{prom}\left(\mathrm{y}_{\mathrm{est}}\right)$ son los promedios correspondientes. Este coeficiente se comparó con el valor crítico ro a la significancia de $5 \%$ (ro $=$ $0,878)$. Cuando el coeficiente fue mayor al valor crítico, se consideró aceptable el modelo para pronóstico de cosecha. En caso contrario, se consideró que el modelo no era apto para pronóstico de cosecha.

\section{Resultados y discusión}

\section{Mejor tamaño de muestra}

Se encontró que para los tres modelos obtenidos por cada estado, el valor del error residual (S) más bajo correspondió al de la muestra de 30 años. Ese número de años se consideró como el tamaño óptimo de la muestra.
En las Tablas 1, 2 y 3 se muestran los valores del error residual S, para los mejores modelos correspondientes a los distintos tamaños de muestras usadas.

Este resultado posiblemente sea causado por la diferencia de variabilidad en los distintos períodos. El crecimiento del rendimiento en los primeros años (1951-1970) es más regular que en los años posteriores, como se puede observar en la Fig. 1 para el estado de Colorado. Después del año 1970 hay un mayor incremento en la variabilidad de los rendimientos. Para corroborar que esta diferencia en la variabilidad de los rendimientos influye en el resultado, se revisó los valores obtenidos del estadístico $\mathrm{F}_{\text {año }}$, para los estados de Colorado, Kansas y Texas.

Colorado: $\mathrm{F}_{\text {año }}=13,67101$

Texas: $\quad \mathrm{F}_{\text {año }}=3,114031$

Kansas: $\quad \mathrm{F}_{\text {año }}=3,218778$

Al revisar estos resultados, se notó que en los tres casos: el estadístico $\mathrm{F}_{\text {año }}$ obtenido era mayor al indicado en Tablas: $\mathrm{F}$ (tabla) $=2,428699$, considerando 0,05 como nivel crítico de significancia. Esto revela que la diferencia entre las varianzas de los dos períodos considerados es significativa. La variabilidad del rendimiento en el periodo (1971-1995) es más heterogénea que la variabilidad del rendimiento en el periodo (1956-1970).

\section{Análisis de los modelos obtenidos}

Los modelos obtenidos fueron los correspondientes a las muestras de 30 años. Se comparó el valor F calculado de cada modelo con el que aparece en Tablas al $5 \%$ de significancia. Se encontró en todos los casos que $\mathrm{F}$ (calculado) $>\mathrm{F}$ (tabla), es decir pasaron la prueba $\mathrm{F}$.

Las variables estimadoras año, temperatura de agosto y cuadrado de la temperatura de junio aparecieron en todos los modelos encontrados. Otras variables que más aparecían en los modelos eran los cuadrados de la temperatura de julio, de la temperatura de agosto, de la precipitación de abril y de la precipitación de mayo. El modelo del estado de Kansas contiene estas variables, además de las temperaturas de junio y julio, la precipitación de junio y el cuadrado de la precipitación de

Tabla 1. Obtención del tamaño óptimo de la muestra - 30 años

\begin{tabular}{ccccccccccc}
\hline estado & Colorado & Dakota Sur & Illinois & Indiana & Iowa & Kansas & Minne-sota & Missouri & Ohio & Texas \\
\hline $\mathbf{S ~ ( k g / h a )}$ & 158,20 & 316,38 & 402,48 & 322,47 & 313,69 & 238,35 & 279,07 & 348,54 & 216,99 & 350,25 \\
\hline
\end{tabular}

Tabla 2. Obtención del tamaño óptimo de la muestra - 35 años

\begin{tabular}{|c|c|c|c|c|c|c|c|c|c|c|}
\hline estado & Colorado & Dakota Sur & Illinois & Indiana & Iowa & Kansas & Minne-sota & Missouri & Ohio & Texas \\
\hline $\mathrm{S}$ (kg/ha) & 240,79 & 483,65 & 581,72 & 366,14 & 420,23 & 387,22 & 434,13 & 527,72 & 371,65 & 588,56 \\
\hline
\end{tabular}

Tabla 3. Obtención del tamaño óptimo de la muestra - 40 años

\begin{tabular}{ccccccccccc}
\hline estado & Colorado & Dakota Sur & Illinois & Indiana & Iowa & Kansas & Minne-sota & Missouri & Ohio & Texas \\
\hline S (kg/ha) & 551,12 & 515,91 & 578,28 & 462,97 & 478,40 & 402,89 & 722,49 & 624,27 & 455,67 & 688,35 \\
\hline
\end{tabular}


junio. La ecuación para el modelo del estado de Kansas es:

rend $=-523183+240$ año -3808 Tjunio -994 Tjulio

+8949 Tagosto -11.5 Pjunio +82.6 (Tjunio) $^{2}+14.8$

$(\text { Tjulio })^{2}-173$ (Tagosto) $)^{2}-0.0273(\text { Pabril })^{2}-0.0199$ $(\text { Pmayo })^{2}+0.0490$ (Pjunio) $^{2}$.

Con rend se denota al rendimiento anual del cultivo, con Tmes al valor promedio mensual de la temperatura de dicho mes, con Pmes se denota la precipitación acumulada en dicho mes, con TTmes al cuadrado del valor promedio mensual de la temperatura de dicho mes y con PPmes al cuadrado de la precipitación acumulada en dicho mes.

Las temperaturas promedio de junio y de agosto están dentro del rango óptimo para el cultivo de maíz (21,2$24^{\circ} \mathrm{C}$, según Benacchio, S., Cañizales, R., Bejarano, A., Avilan, W. y Canchica, W., 1988) en gran parte de los estados. Para los estados de Iowa, Illinois, Indiana y Ohio, las dos variables climáticas están en el rango óptimo. Para los estados de Dakota del Sur y Colorado, la temperatura de agosto está dentro del rango óptimo y para los estados de Kansas y Missouri, la temperatura de junio está dentro del rango óptimo.

La precipitación total de mayo está entre las mayores precipitaciones en gran parte de los estados. Para los estados de Iowa, Ohio, Missouri, Dakota del Sur y Kansas, esta variable climática está entre las tres mayores precipitaciones totales. Para los estados de Colorado y Texas, mayo es el mes de mayor precipitación acumulada.

Dale, R., Nelson, W. y Mc Garraham, J. (1983), en su estudio para el estado de Indiana, presenta en todos sus modelos a la variable temperatura promedio de agosto, y en un grupo de modelos, a la variable cuadrado de la temperatura de junio. Ambas son las variables climáticas

Tabla 3. Modelo del Estado de Colorado

\begin{tabular}{cc}
\hline Variable Estimadora & $\mathrm{P}$ \\
\hline Constante & 0,000 \\
Año & 0,000 \\
Tabril & 0,000 \\
Tmayo & 0,000 \\
Tjunio & 0,002 \\
Tjulio & 0,002 \\
Tagosto & 0,001 \\
Pmayo & 0,001 \\
Pagosto & 0,001 \\
Psetiembre & 0,000 \\
TTabril & 0,000 \\
TTmayo & 0,000 \\
TTjunio & 0,002 \\
TTsetiembre & 0,000 \\
PPmayo & 0,000 \\
PPagosto & 0,000 \\
\hline
\end{tabular}

que más aparecen en los modelos presentados en este trabajo. En cambio, en el estudio de Nelson y Dale (1978) para el estado de Indiana, estas mismas variables climáticas no aparecen en sus modelos.

Por otro lado, ambas referencias presentan en la mayoría de sus modelos a las variables de precipitación total de julio, cuadrado de la precipitación total de julio y temperatura promedio de julio. Las tres variables climáticas aparecen

Tabla 4. Modelo del Estado de Dakota del Sur

\begin{tabular}{cc}
\hline Variable Estimadora & $\mathrm{P}$ \\
\hline Constante & 0,000 \\
Año & 0,000 \\
Tjunio & 0,006 \\
Tjulio & 0,130 \\
Tagosto & 0,000 \\
Tsetiembre & 0,260 \\
Toctubre & 0,038 \\
Pmayo & 0,030 \\
Pjulio & 0,001 \\
Pagosto & 0,001 \\
Psetiembre & 0,002 \\
Poctubre & 0,002 \\
TTjunio & 0,005 \\
TTjulio & 0,164 \\
TTagosto & 0,000 \\
TTsetiembre & 0,192 \\
PPjunio & 0,079 \\
PPagosto & 0,012 \\
PPsetiembre & 0,014 \\
\hline
\end{tabular}

Tabla 5. Modelo del Estado de Illinois

\begin{tabular}{cc}
\hline Variable Estimadora & $\mathrm{P}$ \\
\hline Constante & 0,000 \\
Año & 0,000 \\
Tabril & 0,004 \\
Tagosto & 0,041 \\
Tsetiembre & 0,276 \\
Pabril & 0,008 \\
Pagosto & 0,027 \\
TTjunio & 0,012 \\
TTjulio & 0,028 \\
TTagosto & 0,030 \\
TTsetiembre & 0,303 \\
PPabril & 0,002 \\
PPmayo & 0,093 \\
PPjunio & 0,006 \\
PPsetiembre & 0,023
\end{tabular}


Tabla 6. Modelo del Estado de Indiana

\begin{tabular}{cc}
\hline Variable Estimadora & $\mathrm{P}$ \\
\hline Constante & 0,000 \\
Año & 0,000 \\
Tabril & 0,059 \\
Tmayo & 0,035 \\
Tjunio & 0,223 \\
Tjulio & 0,008 \\
Tagosto & 0,054 \\
Tsetiembre & 0,001 \\
Pabril & 0,033 \\
Pjunio & 0,003 \\
Pagosto & 0,077 \\
Psetiembre & 0,070 \\
TTmayo & 0,032 \\
TTjunio & 0,277 \\
TTjulio & 0,007 \\
TTagosto & 0,037 \\
TTsetiembre & 0,002 \\
PPabril & 0,012 \\
PPagosto & 0,062 \\
\hline
\end{tabular}

Tabla 7. Modelo del Estado de Iowa

\begin{tabular}{cc}
\hline Variable Estimadora & $\mathrm{P}$ \\
\hline Constante & 0,000 \\
Año & 0,000 \\
Tmayo & 0,045 \\
Tjunio & 0,047 \\
Tagosto & 0,003 \\
Tsetiembre & 0,018 \\
Pabril & 0,001 \\
Pjunio & 0,059 \\
Pagosto & 0,030 \\
Psetiembre & 0,056 \\
TTabril & 0,207 \\
TTmayo & 0,042 \\
TTjunio & 0,046 \\
TTjulio & 0,098 \\
TTagosto & 0,002 \\
TTsetiembre & 0,020 \\
PPabril & 0,001 \\
PPmayo & 0,050 \\
PPjunio & 0,042 \\
PPagosto & 0,113 \\
\hline
\end{tabular}

poco en los modelos obtenidos en este trabajo.

A continuación, en las Tablas de la 3 a la 12, se indican las variables estimadoras obtenidas en cada modelo y su significancia con el valor-p. Si p es menor a 0,05 , es significativo al $5 \%$, si p es menor que 0,01 , es significativo al $1 \%$.

La variable estimadora año es significativa al $1 \%$ en todos los modelos obtenidos. Las variables estimadoras, temperatura de mayo, precipitación de abril, el cuadrado de la temperatura de agosto y el cuadrado de la precipitación de abril, se presentaron significativas en todos los modelos en que aparecían. Las otras variables que mayormente se presentaron como significativas fueron la temperatura de agosto y el cuadrado de la

Tabla 8. Modelo del Estado de Kansas

\begin{tabular}{cc}
\hline Variable Estimadora & $\mathrm{P}$ \\
\hline Constante & 0,000 \\
Año & 0,000 \\
Tjunio & 0,012 \\
Tjulio & 0,152 \\
Tagosto & 0,000 \\
Pjunio & 0,005 \\
TTjunio & 0,009 \\
TTjulio & 0,233 \\
TTagosto & 0,000 \\
PPabril & 0,025 \\
PPmayo & 0,000 \\
PPjunio & 0,001 \\
\hline
\end{tabular}

Tabla 9. Modelo del Estado de Missouri

\begin{tabular}{cc}
\hline Variable Estimadora & $\mathrm{P}$ \\
\hline Constante & 0,000 \\
Año & 0,000 \\
Tabril & 0,005 \\
Tjulio & 0,103 \\
Tagosto & 0,002 \\
Tsetiembre & 0,001 \\
Pabril & 0,001 \\
Pjulio & 0,187 \\
Psetiembre & 0,045 \\
TTmayo & 0,003 \\
TTjunio & 0,001 \\
TTjulio & 0,063 \\
TTsetiembre & 0,002 \\
PPabril & 0,000 \\
PPmayo & 0,026 \\
PPjunio & 0,018 \\
PPjulio & 0,267 \\
\hline &
\end{tabular}


Tabla 10. Modelo del Estado de Minnesota

\begin{tabular}{cc}
\hline Variable Estimadora & $\mathrm{P}$ \\
\hline Constante & 0,000 \\
Año & 0,000 \\
Tabril & 0,012 \\
Tmayo & 0,000 \\
Tjulio & 0,019 \\
Tagosto & 0,000 \\
Tsetiembre & 0,010 \\
Pabril & 0,001 \\
Pmayo & 0,000 \\
Psetiembre & 0,003 \\
TTabril & 0,001 \\
TTmayo & 0,001 \\
TTjunio & 0,029 \\
TTjulio & 0,018 \\
TTagosto & 0,000 \\
TTsetiembre & 0,009 \\
PPabril & 0,002 \\
PPmayo & 0,001 \\
PPjulio & 0,001 \\
PPsetiembre & 0,013 \\
\hline &
\end{tabular}

Tabla 11. Modelo del Estado de Ohio

\begin{tabular}{cc}
\hline Variable Estimadora & $\mathrm{P}$ \\
\hline Constante & 0,000 \\
Año & 0,000 \\
Tabril & 0,270 \\
Tmayo & 0,037 \\
Tjunio & 0,175 \\
Tagosto & 0,004 \\
Tsetiembre & 0,007 \\
Pjunio & 0,004 \\
Pjulio & 0,000 \\
Pagosto & 0,037 \\
TTmayo & 0,026 \\
TTjunio & 0,193 \\
TTagosto & 0,002 \\
PPabril & 0,002 \\
PPmayo & 0,140 \\
PPagosto & 0,002 \\
PPsetiembre & 0,000 \\
\hline
\end{tabular}

temperatura de junio.

En el modelo del estado de Colorado, todas las variables son significativas al $1 \%$. En el modelo del estado de Minnesota, todas las variables son significativas, la mayoría al $1 \%$, las otras al $5 \%$. En todos los demás estados, salvo Iowa e Illinois, las variables significativas en su mayoría corresponden al $1 \%$, las otras al $5 \%$.

\section{Modelos aceptables para pronóstico de cosecha}

Se encontró que en la prueba del coeficiente de correlación, el valor obtenido era mayor al valor crítico al $5 \%$ de significancia, solo en el modelo del estado de Indiana ( $\mathrm{r}$ $=0,95)$; en todos los demás modelos, el coeficiente de correlación obtenido era menor que el valor crítico, es decir solo el modelo para el estado de Indiana es aplicable para estimar el rendimiento de maíz en los cinco años siguientes.

Tabla 12. Modelo del Estado de Texas

\begin{tabular}{cc}
\hline Variable Estimadora & $\mathrm{P}$ \\
\hline Constante & 0,000 \\
Año & 0,000 \\
Tmarzo & 0,001 \\
Tabril & 0,010 \\
Tmayo & 0,039 \\
Tjunio & 0,018 \\
Tagosto & 0,001 \\
Pabril & 0,000 \\
Pmayo & 0,023 \\
Pjulio & 0,009 \\
Pagosto & 0,049 \\
TTmarzo & 0,002 \\
TTmayo & 0,051 \\
TTjunio & 0,019 \\
TTjulio & 0,014 \\
TTagosto & 0,001 \\
PPmarzo & 0,004 \\
PPabril & 0,000 \\
PPmayo & 0,094 \\
PPjulio & 0,003 \\
PPagosto & 0,033 \\
\hline
\end{tabular}

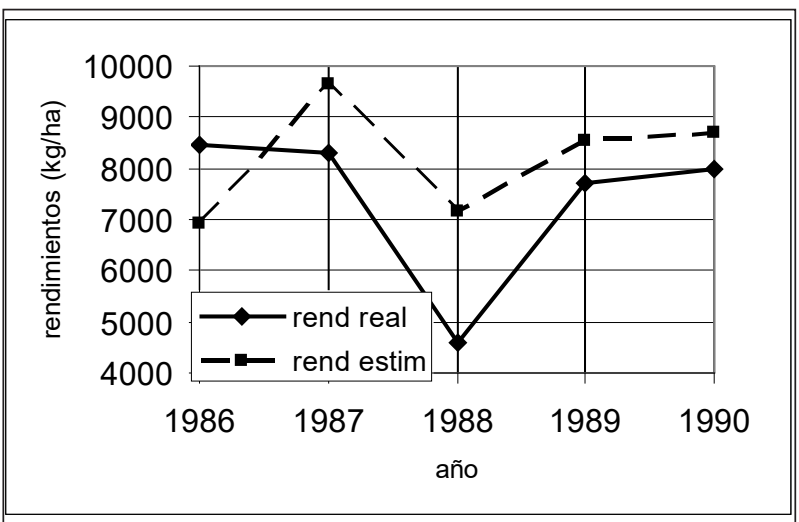

Figura 3. Comparación de los rendimientos real y estimado para los cinco años siguientes a los utilizados para armar los modelos en el estado de Indiana 
En los gráficos de rendimientos estimado y real para los cinco años posteriores al período de la muestra óptima, se observó que en la mayoría de los casos, el rendimiento previsto sobreestimaba el rendimiento real. En la Fig. 3 se muestra el gráfico correspondiente al estado de Indiana. Se puede notar que las evoluciones de los rendimientos real y estimado siguen tendencias parecidas. Tanto el rendimiento real como el rendimiento estimado presentan un mínimo en el año 1988.

\section{Conclusiones}

El tamaño óptimo de la muestra fue de 30 años para todos los estados, es decir la cantidad de observaciones para obtener el mejor modelo de cada estado era de 30 .

Las variables independientes que aparecían en las ecuaciones de todos los modelos obtenidos son el año, la temperatura promedio de agosto y el cuadrado de la temperatura de junio. El análisis estadístico de estos modelos demostró que pasaron la prueba $\mathrm{F}$.

Solo el modelo del Estado de Indiana puede ser utilizado para estimar el rendimiento de maíz en los cinco años siguientes, pues solo en este caso el coeficiente de correlación $r$ de la regresión entre el rendimiento estimado contra el rendimiento observado era mayor que el valor crítico, con significancia al $5 \%$. Los demás modelos no son aplicables para la misma estimación.

\section{Literatura citada}

Benacchio, S.; Cañizales, R.; Bejarano, A.; Avilan, W.y Canchica, W. 1988. Evaluación de Híbridos Zonificación Agroecológica del Cultivo de Maíz (Zea mays L.) en el País. FONAIAP-INIA, Serie C (10-26). Ministerio del Poder Popular Para la Agricultura y Tierras, Venezuela. Disponible en http://www.ceniap.gov.ve/pbd/index.htm

Córdova, N. 2008. Estadística Aplicada. (1ra ed.). Lima, Perú: Editorial Moshera SRL.

Dale, R.; Nelson, W. y Mc Garraham, J. 1983. Effect of Bias in Divisional and State Mean Temperaturas on Wheather-Crop Yield Model Predictions: A Case Study in Indiana. Journal of Climate and Applied Meteorology, 22: 1842-1852.

Molinero. 2002. Construcción de Modelos de Regresión Multivariante. Asociación de la Sociedad Española de Hipertensión. Liga Española para la lucha contra la Hipertensión Arterial. Recuperado de www.seh-lelha. org/pdf/regresion1.pdf

Nelson, W. y Dale, R. 1978. Effect of Trend or Technology Variables and Record Period on Prediction of Corn Yields with Weather Variables. Journal of Applied Meteorology, 17: 926-933.

Snedecor, G. y Cochran, W. 1980. Métodos Estadísticos. (7ma ed). D.F., México: Compañía Editorial Continental, S.A: 